\title{
Manifestações psicossomáticas associadas à síndrome de burnout referidas por trabalhadores de saúde
}

\author{
Pollyana Bortholazzi Gouvêa Maria do Carmo Lourenço Haddad² Mariana Angela Rossaneis ${ }^{3}$
}

\begin{abstract}
RESUMO
O trabalho na área da saúde tem causado grande desgaste físico e psicológico aos trabalhadores. Estes passam a desenvolver mecanismos de defesa manifestados através de reações físicas e psíquicas, acarretando o surgimento de novas enfermidades. Realizou-se um estudo descritivo e quantitativo com trabalhadores que atuam em um hospital de média complexidade. 0 objetivo foi descrever os sintomas físicos relatados pelos trabalhadores que apresentaram sinais da Síndrome de Burnout. Participaram da pesquisa 160 profissionais. Observou-se que todos que apresentavam sinais e sintomas da Síndrome de Burnout relataram a presença de sintomas físicos, sendo os mais frequentes: cefaleias, sentimento de cansaço mental, alterações de apetite, hipertensão e dificuldades com o sono. Estes sintomas podem ser graves e ter grande impacto negativo na vida pessoal e profissional dos portadores da síndrome. 0 diagnóstico precoce da Síndrome de Burnout possibilita uma intervenção mais rápida e eficaz.
\end{abstract}

Descritores: Esgotamento profissional, Saúde do Trabalhador, Manifestações Neurocomportamentais.

\section{Psychosomatic manifestations associated with burnout syndrome referred to by health workers}

\begin{abstract}
The work in the area of health has caused great physical and psychological workers who go on to develop defense mechanisms manifested by physical and psychological reactions, leading to the emergence of new diseases. We conducted a descriptive study with quantitative and workers who work in a hospital of medium complexity. The objective was to describe the physical symptoms reported by workers who had signs and symptoms of burnout syndrome. 160 professionals participated in the survey. It was observed that all who had signs and symptoms of burnout syndrome reported the presence of physical symptoms were the most common: headache, feeling of mental fatigue, changes in appetite, hypertension, and sleep difficulties. These symptoms may be severe and have a great impact on both the professional and personal lives of his victims. It is important to identify these symptoms early to ensure a more rapid and effective.
\end{abstract}

Descriptors: Burnout, Occupational Health, Neurobehavioral Manifestations.

\footnotetext{
${ }^{1}$ Mestre em cirurgia pela Pontifícia Universidade Católica do Paraná (PUC/PR), Curitiba, PR, Brasil.

${ }^{2}$ Doutorado em egenharia elétrica pela Universidade Estadual de Campinas (UNICAMP), Campinhas, SP, Brasil.
} 


\section{Introdução}

A Síndrome de Burnout é caracterizada como uma resposta ao estresse laboral crônico que surge quando o indivíduo não consegue elaborar estratégias de enfrentamento aos agentes estressores ${ }^{1}$.

Diante de um agente estressor existem dois mecanismos de ação do organismo: enfrentamento ou fuga. A partir do momento que o organismo se ativa para fazer frente ao agente estressor, é desencadeada uma série de reações físicas que envolvem o corpo humano, tais como: aceleração dos batimentos cardíacos, aumento do tônus muscular e cardiovascular, com consequente aumento da pressão vascular. Há também um aumento da capacidade de atenção e aceleração de pensamentos, por fim, o individuo realiza uma análise da situação e escolhe qual será a melhor alternativa, que pode ser de enfrentamento ou retirada².

0 processo de estresse é distinguido em três fases: reação de alarme que é quando o organismo é exposto ao agente estressor e coloca suas defesas em funcionamento, se o organismo percebe que o estressor é inofensivo 0 corpo retorna ao seu estado inicial. Porém quando o estressor persiste passa-se a segunda fase que é a etapa de resistência, nesta o organismo tenta adaptar-se a situação. Entretanto, quando o estressor ainda permanece, entra em curso a terceira fase que é a etapa de esgotamento, caracterizada pelo reinício dos sintomas da fase de alarme com consequente desgaste e deterioração do organismo. Ressalta que os agentes estressores podem ser elementos físicos (ruídos, frio, calor, drogas, etc.), cognitivos (vivência de um assalto, discussões, etc.) ou emocionais (medo, sentimento de perda, mudanças, etc. $)^{2}$.

Novas configurações organizacionais têm demandado, em diferentes graus e por entre os diversos setores produtivos, novas exigências de qualidade na execução das tarefas, mais qualificação e novas competências do trabalhador. A necessidade de se adaptar a essa realidade provocou sucessivas mudanças no ambiente de trabalho, o que têm interferido na saúde do trabalhador que passa a desenvolver mecanismos de defesa manifestados através de reações físicas e psíquicas, acarretando o surgimento de novas enfermidades ${ }^{3}$. Assim, é possível afirmar que a saúde mental dos trabalhadores pode estar relacionada com aspectos do trabalho 4 .

O termo burn significa queima e out sugere que a pessoa com esse tipo de estresse tem um desgaste tanto físico quanto emocional ${ }^{5}$. Para melhor entendimento, Burnout é definido por uma tríade de dimensões: a primeira é a exaustão emocional, que se caracteriza pela falta de energia e entusiasmo, sensação de esgotamento, sentimento de frustração e tensão nos trabalhadores; a segunda é a despersonalização, que se entende pelo desenvolvimento de uma insensibilidade emocional, fazendo com que o profissional trate os usuários, colegas e a organização de maneira desumanizada; e a terceira é a baixa realização profissional demonstrada por uma tendência do trabalhador se avaliar de forma negativa, tornando-se infeliz e insatisfeito ${ }^{6}$.

Vários autores ${ }^{2,7}$ referem que a ocorrência de Burnout está frequentemente relacionada com os profissionais que desempenham atividades que os mantém em contato direto com os usuários, prejudicando a qualidade do serviço bem como a qualidade de vida do trabalhador.

Neste contexto, a enfermagem também acompanhou as novidades do mundo do trabalho, já que os profissionais da enfermagem são os que mantêm maior contato com o paciente hospitalizando e também com seus familiares, vivenciando, juntos, situações estressantes. Entretanto, parte destes profissionais não está psicologicamente preparada para lidar com estas situações ${ }^{7}$.

Observa-se que o trabalho na área da saúde tem causado um grande desgaste físico e psicológico aos trabalhadores. Estes, na maioria das vezes, não sabem identificar o que está acontecendo e em decorrência da sobrecarga de trabalho e do sofrimento psíquico podem apresentar doenças como hipertensão arterial, diabetes mellitus, distúrbios ortopédicos, neurológicos, gástricos e psicológicos ${ }^{8}$.

Os principais sintomas apresentados no processo do burnout são subdivididos na obra de Pereira², fundamentado na literatura de diversos autores. Essa subdivisão se apresenta como sintomas físicos, psíquicos, comportamentais e defensivos. Sendo que os sintomas físicos são descritos como fadiga constante, dores musculares, distúrbios do sono, cefaleias, enxaquecas, problemas gastrointestinais, transtornos cardiovasculares, desordens do sistema respiratório, disfunções sexuais e alterações menstruais nas mulheres. Já os psíquicos são: falta de atenção, alterações de memória, lentificação do pensamento, alienação, sentimento de solidão, impaciência, depressão, baixa auto estima, desconfiança, paranóia, dentre outros. Os sintomas comportamentais são assinalados pela negligência, irritabilidade, agressividade, não aceitação de mudanças, falta de iniciativa, comportamentos de risco, 
suicídio, etc.. Por fim, os sintomas defensivos são caracterizados pela tendência ao isolamento, perda do interesse pelo trabalho, absenteísmo, ironia e cinismo².

Diante do exposto, percebe-se que ao estudar as manifestações do burnout entre trabalhadores de saúde, permite-se interpretar algumas dificuldades vivenciadas no processo de trabalho das instituições de saúde, como a insatisfação profissional, a baixa produtividade do trabalho, o absenteísmo, as doenças ocupacionais, dentre outras.

Portanto este estudo tem como objetivo descrever os sintomas físicos relatados por trabalhadores de saúde que apresentaram sinais e sintomas da Síndrome de Burnout.

\section{Materiais e método}

Trata-se de um estudo descritivo de natureza quantitativa. A população do estudo foi constituída por trabalhadores de diversas categorias profissionais que atuam em um hospital de média complexidade de Londrina-PR. Foram convidados para participar do estudo todos os profissionais que estavam ativos em suas funções no momento da coleta de dados, sendo excluídos apenas aqueles que se recusaram a participar da pesquisa ou que estavam em algum tipo de licença. Assim, a população do estudo correspondeu a 136 profissionais de nível superior, técnico e auxiliares operacionais.

O local do estudo é uma instituição pública constituída por pronto socorro, centro cirúrgico e setor de internação clínica e cirúrgica. Este hospital possui 65 leitos de internação e o pronto-socorro funciona 24 horas e atente pacientes encaminhados pelo Serviço de Atendimento Móvel de Urgência (SAMU), Serviço Integrado de Atendimento ao Trauma em Emergência (SIATE), Unidades Básicas de Saúde (UBS) e demanda espontânea.

A instituição encontra-se na região norte do município de Londrina e é referência para o atendimento de uma população de aproximadamente 106 mil pessoas.

Para coleta de dados foi utilizado o instrumento autoaplicável contendo questões para a caracterização sócio ocupacional da população e o questionário Maslach Burnout Inventory (MBI). O MBI é composto por 22 itens que verificam a presença de sintomas que relacionados às três dimensões que caracterizam a Síndrome de Burnout (exaustão emocional, despersonalização e baixa realização profissional). Esse instrumento foi desenvolvido por Christine Maslach, professora universitária de psicologia da Califórnia nos Estados Unidos da América e validado para uso no Brasil em 19864.

O instrumento validado por Pereira ${ }^{2}$ organiza os sintomas da síndrome de burnout em quatro categorias: físicos (fadiga constante e progressiva, distúrbios do sono, dores musculares ou osteomusculares, cefaleia, enxaquecas, perturbações gastrointestinais, imunodeficiência, transtornos cardiovasculares, distúrbios do sistema respiratório, disfunções sexuais e alterações menstruais nas mulheres), psíquicos (falta de atenção e concentração, alterações de memória, alienação, impaciência, labilidade emocional, desânimo), comportamentais (irritabilidade, aumento do consumo de substâncias, incapacidade de relaxar, hiperatividade) e defensivos (isolamento, absenteísmo, perda de interesse pelo trabalho) ${ }^{2}$. 0 presente estudo aborda os sintomas físicos identificados nos participantes que apresentaram à síndrome de burnout.

Os dados foram coletados em 2009, tabulados e analisados utilizando-se o programa Epi Info versão 3.3.2 de 2005 e do software SPSS (Statistical Package for Social Science for Windows versão 13.0), resultando em análise descritiva e cruzamento entre as variáveis pelo teste de Qui Quadrado, com nível de significância de 5\%.

O projeto de pesquisa foi aprovado pelo Comitê de Ética em Pesquisa Envolvendo Seres Humanos da Universidade Estadual de Londrina, registrado sob o CAAE n²931.0.268000-7.

\section{Resultados}

Participaram do estudo 160 profissionais, correspondendo a 56,9\% do quadro de funcionários da instituição.

Para análise dos dados os profissionais foram subdivididos por setores, e somente na área de enfermagem foram separados os profissionais de nível superior (enfermeiros) dos de nível médio e técnico (auxiliares e técnicos de enfermagem). A Tabela 1 apresenta esta distribuição dos profissionais. 
Tabela 1 - Distribuição dos trabalhadores de saúde de uma instituição de média complexidade segundo o setor de trabalho, Londrina-PR, 2009.

\begin{tabular}{|c|c|c|}
\hline Categoria Profissional/Setores & $\mathrm{N}$ & $\%$ \\
\hline Enfermeiros & 08 & 5,0 \\
\hline Aux./Técnicos de enfermagem & 53 & 33,2 \\
\hline Comissão de controle de infecção hospitalar & 03 & 1,9 \\
\hline Costura & 01 & 0,6 \\
\hline Almoxarifado & 02 & 1,3 \\
\hline Apoio a diretoria & 02 & 1,3 \\
\hline Cozinha & 10 & 6,3 \\
\hline Centro de processamento de dados & 01 & 0,6 \\
\hline Departamento pessoal & 02 & 1,3 \\
\hline Diretoria administrativa & 04 & 2,5 \\
\hline Farmácia & 04 & 2,5 \\
\hline Faturamento & 02 & 1,3 \\
\hline Laboratório de análises clínicas & 16 & 10,0 \\
\hline Internação hospitalar & 03 & 1,9 \\
\hline Portaria & 05 & 3,2 \\
\hline Raio X & 06 & 3,8 \\
\hline Registro & 04 & 2,5 \\
\hline Secretários de enfermagem & 02 & 1,3 \\
\hline Serviço Social & 03 & 1,9 \\
\hline Telefonia & 03 & 1,9 \\
\hline Vigilantes & 06 & 3,8 \\
\hline Serviços gerais & 20 & 12,5 \\
\hline Total & 160 & 100 \\
\hline
\end{tabular}

Fonte: autor

A maioria dos participantes da pesquisa foram de profissionais de enfermagem (39,7\%), somando-se enfermeiros, técnicos/auxiliares de enfermagem e profissionais da $\mathrm{CCIH}$, esses exclusivos da área de enfermagem, deste modo obteve-se 64 participantes.

Realizou-se também a caracterização dos participantes da pesquisa de acordo com as variáveis sócio ocupacionais como: idade, gênero, número de filhos, vinculo empregatício dentre outras, com o intuito de obter maior compreensão do perfil dos participantes e com isso identificar se algumas dessas variáveis influenciavam ou estavam relacionadas à presença de sintomas físicos.

De acordo com os dados apresentados na Tabela 2, observou-se que a maioria dos participantes da pesquisa eram mulheres $(67,5 \%)$, com idade entre 31 a 50 anos $(55 \%)$, casadas $(64,4 \%)$, com filhos $(76,9 \%)$ e com apenas um vinculo empregatício $(76,3 \%)$.

Tabela 2 - Caracterização da população do estudo de acordo as variáveis sócio ocupacionais. Londrina-PR, 2009.

\begin{tabular}{c|c|c}
\hline Variáveis sócio ocupacionais & $\mathrm{N}$ & $\%$ \\
\hline Idade & & \\
\hline Entre 20 a 30 anos & 31 & 19,4 \\
\hline Entre 31 a 50 anos & 88 & 55,0 \\
\hline Entre 51 a 65 anos & 38 & 23,7 \\
\hline Mais de 65 anos & 3 & 1,8 \\
\hline
\end{tabular}




\begin{tabular}{|c|c|c|}
\hline Estado Civil & & \\
\hline Casado & 104 & 64,4 \\
\hline Solteiro & 36 & 22,5 \\
\hline União estável & 1 & 0,6 \\
\hline Divorciado & 13 & 8,1 \\
\hline Viúvo & 7 & 4,4 \\
\hline \multicolumn{3}{|l|}{ Sexo } \\
\hline Masculino & 52 & 32,5 \\
\hline Feminino & 108 & 67,5 \\
\hline \multicolumn{3}{|l|}{ Filhos } \\
\hline Sim & 123 & 76,9 \\
\hline Não & 37 & 23,1 \\
\hline \multicolumn{3}{|c|}{ Tipo de Vínculo Empregatício } \\
\hline Estatuário & 74 & 46,2 \\
\hline Celetista & 84 & 52,5 \\
\hline Outro & 2 & 1,2 \\
\hline \multicolumn{3}{|l|}{ Titulação } \\
\hline Ensino fundamental & 39 & 24,4 \\
\hline Ensino médio & 81 & 50,6 \\
\hline Graduação & 21 & 13,1 \\
\hline Especialização & 17 & 10,6 \\
\hline Mestrado & 1 & 0,6 \\
\hline Doutorado & 1 & 0,6 \\
\hline \multicolumn{3}{|l|}{ Outro emprego } \\
\hline Sim & 28 & 23,7 \\
\hline Não & 132 & 76,3 \\
\hline
\end{tabular}

Fonte: autor

Ao relacionar a tríade do burnout com sintomas físicos, encontrou-se que todos que apresentavam alto grau de exaustão emocional, alto grau de despersonalização e baixa realização profissional relataram a presença de sintomas físicos. Os valores de p variaram entre $p=0,000$ a $p=0,030$ ao se aplicar o teste do Qui Quadrado, demonstrando relevância estatística neste estudo.

Na tabela Tabela 3 verificam-se a frequência em que os sintomas físicos foram referidos pelos participantes identificados com exaustão emocional, despersonalização e baixa realização profissional.

Tabela 3 - Frequência em que os sintomas físicos foram referidos pelos participantes identificados com exaustão emocional, despersonalização e baixa realização profissional, Londrina-PR, 2009.

\begin{tabular}{|c|c|c|c|c|c|c|}
\hline \multirow[t]{2}{*}{ Sintomas físicos } & \multicolumn{6}{|c|}{ Dimensões da síndrome de Burnout } \\
\hline & \multicolumn{2}{|c|}{ Exaustão emocional } & \multicolumn{2}{|c|}{ Despersonalização } & \multicolumn{2}{|c|}{ Baixa realização profissional } \\
\hline Cefaleia & 41 & 75,9 & 29 & 67,4 & 34 & 70,8 \\
\hline Alterações do apetite & 35 & 64,8 & 43 & 100,0 & 24 & 50,0 \\
\hline Hipertensão & 25 & 47,2 & 24 & 55,8 & 48 & 100,0 \\
\hline Distúrbios do sono & 37 & 68,5 & 29 & 67,4 & 31 & 63,3 \\
\hline Cansaço Mental & 48 & 88,9 & 37 & 86,0 & 41 & 83,7 \\
\hline Dificuldades sexuais & 27 & 51,9 & 17 & 39,5 & 26 & 53,1 \\
\hline Fadiga & 32 & 59,3 & 28 & 65,1 & 32 & 65,3 \\
\hline Pequenas infecções & 30 & 55,6 & 20 & 46,5 & 22 & 44,9 \\
\hline Problemas gastrointestinais & 34 & 63,0 & 22 & 51,2 & 28 & 57,1 \\
\hline Problemas respiratórios & 29 & 53,7 & 20 & 46,5 & 20 & 40,8 \\
\hline Dores musculares & 48 & 88,9 & 31 & 72,1 & 34 & 69,4 \\
\hline
\end{tabular}

Fonte: autor 
Como demonstrado acima, pode-se observar que alguns sintomas, dentre eles, cefaleias, distúrbios do sono, cansaço mental e dores musculares estiveram presentes em mais da metade (60\%) dos participantes da pesquisa em todas as dimensões da síndrome de burnout. Contudo, nota-se que a sensação de cansaço mental foi o que mais prevaleceu entre os participantes, seguido por dores musculares.

\section{Discussão}

O desenvolvimento do trabalho do homem depende de sua aptidão, habilidade e capacidade de produzir no ambiente em que vive. Neste processo de interação com o meio, mediado por instrumentos fabricados, o homem, modifica e é modificado pela natureza. Dentre as inúmeras modificações, encontram-se aquelas que possuem consequências físicas e psíquicas ${ }^{3}$.

O estresse é uma dessas consequências e encontra-se presente no dia-a-dia das pessoas e frequentemente está associado ao trabalho e as condições de vida, todavia é no trabalho que se ressaltam os sinais de exaustão emocional ${ }^{6}$. Essa exaustão que muitas vezes decorre do trabalho e a própria percepção do desgaste pode ser definida como uma sensação de que o indivíduo está sendo exigido além dos seus limites de resistência ${ }^{9}$.

Nesse sentido, a instituição hospitalar, que apresenta uma estrutura organizacional complexa quanto aos profissionais, papéis, estrutura, divisão de trabalho, metas, hierarquia e normas que a regulam e possuidora de uma prática profissional voltada, quase que exclusivamente para a eficácia do atendimento ao paciente, muitas vezes, percebe-se uma menor valorização das condições de trabalho essenciais para a saúde do trabalhador, que permanecem expostos por um período prolongado a situações que exigem alta demanda física, psíquica e emocional ${ }^{10}$.

Escores elevados de exaustão emocional demonstram o desequilíbrio entre o indivíduo e o trabalho, gerando 0 desgaste físico ${ }^{11}$. A insatisfação com 0 ambiente de trabalho, com a função exercida e com a falta de participação nas tomadas de decisão eleva o sentimento de desgaste emocional e a incidência de doenças psicossomáticas relacionadas ao trabalho ${ }^{12}$.

Os fenômenos psicossomáticos têm, sobretudo, uma função defensiva. Parecem corretas certas observações quanto à dificuldade em identificar emoções, sentimentos e sensações dos envolvidos e essa regressão explicaria o fato de as mensagens enviadas pelo corpo ao psiquismo, ou o inverso, serem inscritas sem representações de palavras e sim por meios físicos ${ }^{13}$.

As fases do estresse possuem processos, reações biológicas e psicossociais específicas. Sendo que na primeira fase (fase de alarme), os sintomas característicos são: alterações cardiovasculares, insônia, sensação de esgotamento, irritabilidade e fadiga. Na segunda fase (fase de resistência), o organismo tenta manter sua homeostase interna e os sintomas evidenciados são: mudança de humor, depressão, isolamento social, alterações no apetite e diminuição do desejo sexual. A terceira e última fase, a de exaustão, é manifestada através do adoecimento do órgão, tendo como sintomas característicos: angústia, depressão, apatia, cansaço excessivo, taquicardia, vontade de fugir ${ }^{14}$.

Neste contexto, observa-se que os sujeitos envolvidos na presente pesquisa transitam entre todas as fases de estresse, somados a isso encontramos também que aqueles com alto grau de exaustão emocional foram os que apresentaram maior porcentagem das manifestações físicas quando comparadas as outras dimensões.

Outros estudos ${ }^{15,16}$ também narram a ocorrência de tais manifestações psicossomáticas em suas populações e ressaltam, ainda, que possivelmente estes sintomas são ligados a síndrome de burnout.

O exercício da profissão de enfermagem requer boa saúde física e mental, porém raramente os enfermeiros recebem os subsídios adequados para o seu desempenho, ou seja, apesar de exercerem atividades estafantes, frequentemente em locais insalubres, não recebem a proteção e atenção necessárias para evitar os acidentes e as doenças decorrentes das atividades ${ }^{3}$.

Sabe-se que a prática de enfermagem ainda é dominada pelo sexo feminino e associado ao exposto nota-se que este estudo também verificou a predominância do mesmo. Outras pesquisas ${ }^{2,16}$ também retratam a mesma predominância de gênero e acrescentam que este é um fator predisponente síndrome de burnout ${ }^{17}$, pois foi observado que as mulheres são mais inclinadas ao estresse. Dentre elas as doenças psicossomáticas são ainda mais frequentes, como a enxaqueca, problemas gastrointestinais e musculares. 
Contudo, as mulheres, embora mais estressadas do que os homens, sabem lidar melhor com o estresse. Isto ocorre por questões culturais, associadas às exigências que lhes são impostas, tais como trabalhar fora e, ao mesmo tempo, assumir responsabilidades domésticas ${ }^{18}$.

A variável idade também está relacionada ao nível de burnout e suas dimensões, conforme estudos realizados sobre o tema ${ }^{10}$. Na literatura, observa-se que profissionais jovens geralmente são os que mais sofrem no ambiente de trabalho, pois muitas vezes, possuem um entendimento irrealístico sobre o que podem e o que não podem fazer, tendo como consequência frequentes frustrações profissionais ${ }^{19}$.

No presente estudo, a faixa etária predominante ficou acima dos 30 anos de idade, demonstrando que os profissionais já possuíam razoável tempo de experiência. Verifica-se que profissionais com maior tempo de profissão, provavelmente já desenvolveram estratégias que lhes permitem lidar de maneira melhor com as situações estressantes advindas do trabalho. Porém, no presente estudo não foi encontrado associação significativa entre idade, tempo de formação e aparecimento de sintomas físicos relacionados ao estresse.

Vale ressaltar que estímulos estressores podem ser potentes o suficiente para desencadear o estresse na grande maioria das pessoas, contudo devem-se considerar as diferentes respostas do indivíduo ou grupo frente a situações que, muitas vezes, apresentam-se como semelhantes.

Os sintomas físicos podem ser graves e ter grande impacto tanto na vida pessoal quanto na profissional de suas vitimas. É importante identificar precocemente esses sintomas para garantir uma intervenção mais adequada e eficaz ${ }^{8}$.

\section{Conclusão}

Nessa pesquisa identificou-se que todos os participantes que apresentavam alto grau de exaustão emocional, alto grau de despersonalização e baixa realização profissional apresentavam de sintomas físicos. A presença de cefaleia, distúrbios do sono, cansaço mental e dores musculares foram às doenças psicossomáticas mais frequentes na população.

A prevenção da síndrome de burnout é a melhor maneira de combater esta patologia entre os trabalhadores. Manter equilíbrio entre vida pessoal e profissional, dedicar tempo à família, relaxar, engajar-se em atividades prazerosas e praticar atividades físicas regularmente possibilitam o alívio de tensões e o combate ao estresse.

As organizações de saúde devem estabelecer programas de saúde do trabalhador que envolvam estratégias de humanização do trabalho e proporcionem apoio psicológico e social aos profissionais

\section{Referências Bibliográficas}

1. Gil-Monte PR, Marucco MA. Prevalencia Del "síndrome de quemarse por El trabajo" (burnout) em pediatras de hospitales generales. Rev Saúde Publica. 2008; 42(3):450-56.

2. Pereira AMB. Burnout: quando o trabalho ameaça o bem-estar do trabalhador. 4ed. São Paulo: Casa do Psicólogo, 2011.

3. Carvalho CG, Magalhães SR. Síndrome de burnout e suas consequências nos profissionais de enfermagem. RUVRD. 2011; 9(1):200-10.

4. Rebouças D, Legay LF, Abelha L. Satisfação com o trabalho e impacto causado nos profissionais de saúde mental. Rev Saúde Publica. 2007; (41)2:244-50.

5. Vieira I. Conceitos de burnout: questões atuais da pesquisa e a contribuição da clínica. Rev bras saúde ocup. 2010, 35(122):269-76.

6. Carlotto MS, Palazzo LS. Sindrome de Burnout e fatores associados: um estudo epidemiológico com professores. Cad. Saúde Publica. 2006, 22(5): 1017-26.

7. Gil-Monte, P \& Peiró, J Ma . Desgaste psíquico en el trabajo: El Sindrome de Quemarse. Madrid: Editorial Sintesis, 1997. 8. Oliveira MIV, Bezerra Filho JG, Sousa AM, Gonçalves RF. Estresse ocupacional: Manifestações de Sintomas Físicos, Psicológicos e Sociais dos Profissionais de Enfermagem de uma Maternidade. Ciencia \& Trabajo. 2012. 14(45):254-59.

9. Tamayo MR, Truccoli BT. Exaustão emocional: relações com a percepção de suporte organizacional e com as estratégias de coping no trabalho. Estud. psicol. (Natal). 2002; 7(1):37-46. 
10. Selmanovic S, Ramic E, Pranjic N, Brekalo-Lazarevic S, Pasic Z, Alic A. Stress at work and burnout syndrome in hospital doctors. Med Arh. 2011; 65(4): 221-4.

11. Rosa C, Carlotto MS. Síndrome de Burnout e satisfação no trabalho em profissionais de uma instituição hospitalar. Ver SBPH. 2005, 8(2):1-15.

12. Capitão, CG, Carvalho EB. Psicossomática: duas abordagens de um mesmo problema. Rev Psic. 2006; 7(2):21-9.

13. Maia MVM, Pinheiro NNB. Angústia e subjetividade: reflexões sobre os fenômenos psicossomáticos a partir de Freud e Winnicott. Rev Mal-Estar Subj. 2009; 9(1):75-104.

14. Almeida NDV. Considerações acerca da incidência do estresse em motoristas profissionais. Rev de Psicologia. 2010; $\mathrm{v}(1): 75-84$.

15. Galindo RH, Feliciano KVO, Lima RAS. Síndrome de Burnout entre enfermeiros de um hospital geral da cidade do Recife. Rev Esc Enferm USP. 2012; 46(2):420-7.

16. Almeida CV, Silva C, Centurion P, Chiuzi RM. Síndrome de Burnout em professores: um estudo comparativo na região do Grande ABC paulista. REGS. 2011, 2(1): 276-291.

17. Santos FE, Alves JA, Rodrigues AB. Síndrome de Burnout em enfermeiros atuantes em uma Unidade de Terapia Intensiva. Einstein. 2009; 7(1):58-63.

18. Ritter RS, Stumm EMF, Kircher RM. Análise de Burnout em profissionais de uma unidade de emergência de um hospital geral. Rev Eletr Enf. 2009; 11(2):236-48.

19. Franco GP, Barros ALBL, Nogueira-Martins LA, Zeitoun SS. Burnout in nursing residents. Rev esc enferm USP. 2011; 45(1):12-18.

\section{Mariana Angela Rossaneis}

Endereço para correspondência - Rua Brasília , n 1100. Bairro Jardim Vale Verde, CEP 86600000, Rolândia, PR, Brasil.

E-mail: marianarossaneis@gmail.com

Currículo Lattes: http://lattes.cnpq.br/4568754472151940

Pollyana Bortholazzi Gouvêa - pollyanagouvea@usp.br

Maria do Carmo Lourenço Haddad - haddad@uel.br

Recebido em 22 de setembro de 2013.

Publicado em 30 de janeiro de 2014. 